\title{
Lovász-Schrijver PSD-operator on some graph classes defined by clique cutsets
}

\author{
Annegret Wagler \\ LIMOS (UMR 6158 CNRS), Université Clermont Auvergne, Clermont-Ferrand, France \\ annegret.wagler@uca.fr
}

February 6, 2019

\begin{abstract}
This work is devoted to the study of the Lovász-Schrijver PSD-operator $L S_{+}$applied to the edge relaxation $\operatorname{ESTAB}(G)$ of the stable set polytope $\operatorname{STAB}(G)$ of a graph $G$. In order to characterize the graphs $G$ for which $\operatorname{STAB}(G)$ is achieved in one iteration of the $L S_{+}$-operator, called $L S_{+}$-perfect graphs, an according conjecture has been recently formulated $\left(L S_{+}\right.$-Perfect Graph Conjecture). Here we study two graph classes defined by clique cutsets (pseudothreshold graphs and graphs without certain Truemper configurations). We completely describe the facets of the stable set polytope for such graphs, which enables us to show that one class is a subclass of $L S_{+}$-perfect graphs, and to verify the $L S_{+}$-Perfect Graph Conjecture for the other class.
\end{abstract}

\section{Introduction}

In this work, we study the stable set polytope, some of its linear and semidefinite relaxations, and graph classes for which certain relaxations are tight.

The stable set polytope $\operatorname{STAB}(G)$ of a graph $G=(V, E)$ is defined as the convex hull of the incidence vectors of all stable sets of $G$ (in a stable set all nodes are mutually nonadjacent). Two canonical relaxations of $\operatorname{STAB}(G)$ are the edge constraint stable set polytope

$$
\operatorname{ESTAB}(G)=\left\{\mathbf{x} \in[0,1]^{V}: x_{i}+x_{j} \leq 1, i j \in E\right\},
$$

and the clique constraint stable set polytope

$$
\operatorname{QSTAB}(G)=\left\{\mathbf{x} \in[0,1]^{V}: x(Q)=\sum_{i \in Q} x_{i} \leq 1, Q \subseteq V \text { clique }\right\}
$$

(in a clique all nodes are mutually adjacent, hence a clique and a stable set share at most one node). We have $\operatorname{STAB}(G) \subseteq \operatorname{QSTAB}(G) \subseteq \operatorname{ESTAB}(G)$ for any graph, where $\operatorname{STAB}(G)$ equals $\operatorname{ESTAB}(G)$ for bipartite graphs, and $\operatorname{QSTAB}(G)$ 
for perfect graphs only [7]. Perfect graphs are precisely the graphs without chordless cycles $C_{2 k+1}$ with $k \geq 2$, termed odd holes, or their complements, the odd antiholes $\bar{C}_{2 k+1}[5]$.

There are several ways to tighten relaxations with the goal to become closer to the integral polytope, here the stable set polytope.

The Chvátal-Gomory procedure is such a method that adds inequalities to linear relaxations, generated on the basis of the constraint system of the studied relaxation, see [6]. If an inequality $\mathbf{a}^{T} \mathbf{x} \leq b$ is valid for a rational polyhedron $P \subset \mathbf{R}^{n}$ and $\mathbf{a} \in \mathbf{Z}^{n}$, then $\mathbf{a}^{T} \mathbf{x} \leq\lfloor b\rfloor$ is valid for the integer polyhedron $P_{I}:=\operatorname{conv}\left(P \cap \mathbf{Z}^{n}\right)$ and called a Chvátal-Gomory cut. The Chvátal closure of a relaxation is the system of all inequalities that can be generated that way. For instance, Chvátal showed in [6] that from the edge constraints defining $\operatorname{ESTAB}(G)$, only one type of inequalities (associated with odd cycles in $G$ ) can be generated and called the graphs $G$ whose stable set polytope is described that way t-perfect.

Lovász and Schrijver introduced in [16] the PSD-operator $L S_{+}$(called $N_{+}$ in [16]) which has the potential to tighten relaxations in a much stronger way. We denote by $\mathbf{e}_{0}, \mathbf{e}_{1}, \ldots, \mathbf{e}_{n}$ the vectors of the canonical basis of $\mathbf{R}^{n+1}$ (where the first coordinate is indexed zero) and by $S_{+}^{n}$ the convex cone of symmetric and positive semi-definite $(n \times n)$-matrices with real entries. Given a convex set $K$ in $[0,1]^{n}$, let

$$
\operatorname{cone}(K)=\left\{\left(\begin{array}{c}
x_{0} \\
\mathbf{x}
\end{array}\right) \in \mathbf{R}^{n+1}: \mathbf{x}=x_{0} \mathbf{y} ; \mathbf{y} \in K\right\} .
$$

Then, we define the polyhedral set

$$
\begin{aligned}
M_{+}(K)=\left\{Y \in S_{+}^{n+1}: \quad\right. & Y \mathbf{e}_{0}=\operatorname{diag}(Y), Y \mathbf{e}_{i} \in \operatorname{cone}(K), \\
& \left.Y\left(\mathbf{e}_{0}-\mathbf{e}_{i}\right) \in \operatorname{cone}(K), i=1, \ldots, n\right\}
\end{aligned}
$$

where $\operatorname{diag}(Y)$ denotes the vector whose $i$-th entry is $Y_{i i}$, for every $i=0, \ldots, n$. Projecting this lifting back to the space $\mathbf{R}^{n}$ results in

$$
L S_{+}(K)=\left\{\mathrm{x} \in[0,1]^{n}:\left(\begin{array}{c}
1 \\
\mathbf{x}
\end{array}\right)=Y \mathbf{e}_{0}, \text { for some } Y \in M_{+}(K)\right\} .
$$

In [16], Lovász and Schrijver proved that $L S_{+}(K)$ is a relaxation of the convex hull of integer solutions in $K$ and that $L S_{+}^{n}(K)=\operatorname{conv}\left(K \cap\{0,1\}^{n}\right)$, where $L S_{+}^{0}(K)=K$ and $L S_{+}^{k}(K)=L S_{+}\left(L S_{+}^{k-1}(K)\right)$ for every $k \geq 1$.

This operator, applied to $\operatorname{ESTAB}(G)$, generates a positive semi-definite relaxation $L S_{+}(G)$ of $\operatorname{STAB}(G)$. Lovász and Schrijver [16] showed that the following class of inequalities is valid for $L S_{+}(G)$ : joined antiweb constraints

$$
\sum_{i \leq k} \frac{1}{\alpha\left(A_{i}\right)} x\left(A_{i}\right)+x(Q) \leq 1
$$

associated with the complete join of some antiwebs $A_{1}, \ldots, A_{k}$ and a clique $Q$. An antiweb $A_{n}^{k}$ is a graph with $n$ nodes $0, \ldots, n-1$ and edges $i j$ if and only if 
$k \leq|i-j| \leq(n-k) \bmod n$. Note that antiwebs include cliques $K_{k}=A_{k}^{1}$, odd holes $C_{2 k+1}=A_{2 k+1}^{k}$ and odd antiholes $\bar{C}_{2 k+1}=A_{2 k+1}^{2}$.

We denote by $\operatorname{ASTAB}^{*}(G)$ the linear relaxation of $\operatorname{STAB}(G)$ given by all joined antiweb constraints and conclude

$$
\operatorname{STAB}(G) \subseteq L S_{+}(G) \subseteq \operatorname{ASTAB}^{*}(G)
$$

as joined antiweb constraints are valid for $L S_{+}(G)$ by [16].

Graphs $G$ with $\operatorname{STAB}(G)=L S_{+}(G)$ are called $L S_{+}$-perfect, and all other graphs $L S_{+}$-imperfect. A conjecture has been proposed in [1], which can be equivalently reformulated as follows, as noted in [13]:

Conjecture 1 ( $L S_{+}$-Perfect Graph Conjecture) $G$ is $L S_{+}$-perfect if and only if $L S_{+}(G)=\operatorname{ASTAB}^{*}(G)$.

Note that graphs $G$ with $\operatorname{STAB}(G)=\operatorname{ASTAB}^{*}(G)$ are called joined a-perfect by [10]. By (2), we have that all joined a-perfect graphs are $L S_{+}$-perfect.

Subclasses of joined a-perfect graphs can be obtained by restricting joined antiweb constraints to special cases. Well-studied subclasses include, besides perfect graphs, t-perfect, h-perfect, and a-perfect graphs, whose stable set polytopes are given by nonnegativity constraints and joined antiweb constraints associated with edges, triangles and odd holes (resp. cliques and odd holes, resp. antiholes). Note that antiwebs are a-perfect by [19]. Besides these polyhedrally defined subclasses, the only known examples of joined a-perfect graphs are near-bipartite graphs (where the non-neighbors of every node induce a bipartite graph) due to Shepherd [17].

Moreover, we can easily see from the above remarks that the conjecture in fact states that $L S_{+}$-perfect graphs coincide with joined a-perfect graphs.

Conjecture 1 has already been verified for several graph classes: fs-perfect graphs [1] (where the only facet-defining subgraphs are cliques and the graph itself), webs [12] (the complements $W_{n}^{k}=\bar{A}_{n}^{k}$ of antiwebs), line graphs [13] (obtained by turning adjacent edges of a root graph into adjacent nodes of the line graph), and claw-free graphs [2]; the latter result includes graphs $G$ with stability number $\alpha(G)$ at most 2 .

Our aim is to verify Conjecture 1 for further graph classes and to identify further subclasses of joined a-perfect and $L S_{+}$-perfect graphs.

For that, we study graph classes where clique cutsets play a role in a decomposition theorem. A clique cutset of a graph $G$ is a (possibly empty) clique $Q$ such that removing $Q$ disconnects $G$. Many graph classes can be characterized as those graphs that either have a clique cutset or belong to some basic families. A famous example is the class of chordal graphs (that are graphs that contain no holes $C_{k}$ with $k \geq 4$ ):

Theorem 2 ([11]) A graph is chordal if and only if it is a clique or has a clique cutset. 
Here we study two graph classes defined in a similar spirit by clique cutsets $Q$ whose blocks of the decomposition (i.e. the subgraphs $G\left[V_{i} \cup Q\right]$ induced by the clique $Q$ and any component $V_{i}$ of $G-Q$ ) belong to some basic families. We describe the facet-defining system of the stable set polytopes for each of those basic families and then apply the result of Chvátal [7] that the facets of $\operatorname{STAB}(G)$ belong to the union of the facets of the stable set polytopes of the blocks of the decomposition.

A generalization of threshold graphs. Given a 0/1-matrix A, Chvátal and Hammer asked in [8] whether there is a single linear inequality $\mathbf{a}^{T} \mathbf{x} \leq b$ whose $0 / 1$-solutions $\mathbf{x}$ are precisely the $0 / 1$-solutions of the system $A \mathbf{x} \leq \mathbf{1}$ with $\mathbf{1}=(1, \ldots, 1)^{T}$. They showed that this is the case if and only if $A$ is a threshold matrix. Furthermore, they studied the intersection graph $G(A)$ of $A$ whose nodes stand for the columns of $A$ where two nodes are adjacent if and only if the corresponding columns of $A$ have a positive scalar product. Then the $0 / 1$-solutions $\mathbf{x}$ of the system $A \mathbf{x} \leq \mathbf{1}$ are precisely the characteristic vectors of stable sets in $G(A)$. Chvátal and Hammer called a graph threshold if and only if it is the intersection graph $G(A)$ of a threshold matrix $A$.

As a generalization of threshold graphs, Chvátal and Hammer [8] call a graph $G=(V, E)$ pseudothreshold if there are real numbers $b$ and $a_{v}$ for all $v \in V$ such that for every subset $V^{\prime} \subseteq V$,

$$
\begin{aligned}
& \sum_{v \in V^{\prime}} a_{v}<b \Rightarrow V^{\prime} \text { is stable } \\
& \sum_{v \in V^{\prime}} a_{v}>b \Rightarrow V^{\prime} \text { is not stable }
\end{aligned}
$$

and characterized pseudothreshold graphs as follows:

Theorem 3 ([8]) A graph $G=(V, E)$ is pseudothreshold if and only if there is a partition $V=S \cup Q \cup U$ such that

- $S$ is stable, and there are no edges between $S$ and $U$,

- $Q$ is a clique, and there are all edges between $Q$ and $U$,

- $U$ does not contain a stable set of size 3 .

That way, pseudothreshold graphs $G=(S \cup Q \cup U, E)$ contain several subclasses, which include

- graphs with stability number $\alpha(G)=2$ (i.e., with $S=\emptyset$ ),

- graphs without induced $C_{4}, \bar{C}_{4}$ (which are by Blázsik et al. [3] precisely the pseudothreshold graphs with $U=\emptyset$ or $\left.U=C_{5}\right)$ ),

- split graphs (i.e., with $U=\emptyset$ by [14]), and

- threshold graphs (without induced $P_{4}, C_{4}, \bar{C}_{4}$ by [8]).

Hence, pseudothreshold graphs contain two subclasses of chordal graphs: threshold graphs and split graphs [14]. 
Moreover, Chvátal and Hammer noted that (3) can be satisfied for any pseudothreshold graph $G=(S \cup Q \cup U, E)$ with $b=2$ and

$$
a_{v}=\left\{\begin{array}{lll}
0 & \text { if } & v \in S \\
1 & \text { if } & v \in U \\
2 & \text { if } & v \in Q
\end{array}\right.
$$

Indeed, $a\left(V^{\prime}\right)<2$ if $V^{\prime}$ is a subset $S^{\prime}$ of $S$, or equals $S^{\prime} \cup\{u\}$ for some $u \in U$, but we have $a\left(V^{\prime}\right)>2$ if $V^{\prime}$ contains 3 nodes from $U$, or one node from $U$ and $Q$ each, or two nodes from $Q$. However, for other subsets $V^{\prime}$, e.g. $\left\{u, u^{\prime}\right\} \subseteq U$ or $S^{\prime} \cup\{q\}$ for some $q \in Q$, it is not decidable via (3) whether or not $V^{\prime}$ is stable.

We provide the system of linear inequalities that exactly encodes all characteristic vectors of stable sets of a pseudothreshold graph. That is, we will present the facet-defining inequalities of the stable set polytope $\operatorname{STAB}(G)$ for $G$ pseudothreshold. As a consequence, we can verify the $L S_{+}$-Perfect Graph Conjecture for pseudothreshold graphs.

Moreover, we define a graph $G$ to be strongly pseudothreshold if both $G$ and its complement $\bar{G}$ are pseudothreshold, and show that strongly pseudothreshold graphs are joined a-perfect (see Section 2).

Generalizations of chordal graphs. A graph $G$ is universally signable if for every prescription of parities to its holes, there exists an assignment of $0 / 1$ weights to its edges such that for each hole, the weights of its edges sum up to the prescribed parity, and for each triangle, the sum of the weights of its edges is odd. Truemper [18] studied universally signable graphs and identified the following forbidden configurations for such graphs:

- thetas (subdivisions of the complete bipartite graph $K_{2,3}$ ),

- pyramids (subdivisions of the complete graph $K_{4}$ such that one triangle remains unsubdivided),

- prisms (subdivisions of $\bar{C}_{6}$ where the two triangles remain unsubdivided),

- wheels (consisting of a hole $C$ and an additional node $v$ having at least 3 neighbors on $C$ ).

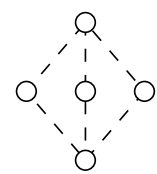

(a)

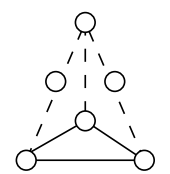

(b)

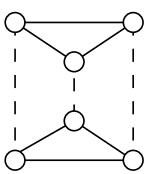

(c)

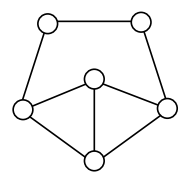

(d)

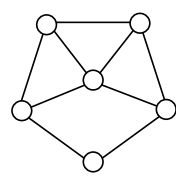

(e)

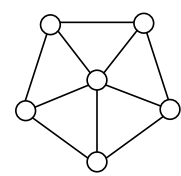

(f)

Figure 1: Truemper configurations: (a) thetas, (b) pyramids, (c) prisms, (d)-(f) wheels (in the drawings, a full line represents an edge, a dashed line a path).

Conforti et al. [9] called them Truemper configurations (see Fig. 1) and characterized universally signable graphs as follows: 
Theorem 4 ([9]) A graph is universally signable if and only if it has no Truemper configuration. A universally signable graph is either a clique, a hole, or has a clique cutset.

Thus, universally signable graphs form a superclass of chordal graphs. Boncompagni et al. [4] defined further superclasses of universally signable graphs by allowing some wheels as induced subgraphs.

A wheel is called universal if the additional node $v$ is adjacent to all nodes of $C$, a twin wheel if $v$ is adjacent to precisely 3 consecutive nodes of $C$, and proper otherwise (see Fig. 1(d)-(f) for examples). Note that universal wheels are often just called wheels in the literature and are, in fact, the complete join of a single node and a hole.

$\mathcal{G}_{U}$ denotes the class of all graphs not having thetas, pyramids, prisms, proper wheels and twin wheels. Hence, the only Truemper configurations that can occur in graphs in $\mathcal{G}_{U}$ are universal wheels.

Boncompagni et al. provided a decomposition result for $\mathcal{G}_{U}$ in terms of clique cutsets and identified two basic families:

- graphs $G$ such that every anticomponent ${ }^{1}$ of $G$ is isomorphic to $K_{1}$ or $\bar{K}_{2}$; we call such graphs light cliques as they can be obtained from a clique by removing a (possibly empty) matching (and note that they are perfect);

- graphs $G$ such that one anticomponent of $G$ is a hole $C_{k}$ with $k \geq 5$ and all other anticomponents of $G$ (if any) are isomorphic to $K_{1}$; we call such graphs fat universal wheels as they can be obtained as complete join of the hole $C_{k}$ and a (possibly empty) clique.

Using these terms, the decomposition result of Boncompagni et al. reads as follows:

Theorem 5 ([4]) Every graph in $\mathcal{G}_{U}$ is either a light clique, a fat universal wheel, or has a clique cutset.

Based on this result, we give a complete description of the stable set polytope for graphs in $\mathcal{G}_{U}$ and conclude that every graph in $\mathcal{G}_{U}$ is joined a-perfect (see Section 3).

Finally, we discuss the relations of the studied graph classes, revealing that strongly pseudothreshold graphs form a subclass of $\mathcal{G}_{U}$ and that $\mathcal{G}_{U}$ is a new subclass of joined a-perfect graphs, being incomparable to all such classes known so far. We close with some concluding remarks.

\section{About pseudothreshold graphs}

In order to describe the facet-defining inequalities of the stable set polytope of pseudothreshold graphs, we rely on the following results from the literature.

\footnotetext{
${ }^{1} \mathrm{An}$ anticomponent is an inclusion-wise maximal subgraph $G^{\prime}$ of $G$ such that $\bar{G}^{\prime}$ is a component of $\bar{G}$.
} 
Recall that a pseudothreshold graph has a partition of its node set into $S \cup Q \cup U$ by Theorem 3 .

A pseudothreshold graph $G=(S \cup Q, E)$, i.e. with $U=\emptyset$, is a split graph and thus perfect by [14], so its stable set polytope is given by nonnegativity and clique constraints only [7].

A pseudothreshold graph $G=(U \cup Q, E)$, i.e. with $S=\emptyset$, has $\alpha(G) \leq 2$ by Theorem 3. Cook (see [17]) studied the stable set polytope of graphs $G$ with $\alpha(G)=2$ and showed that only the following type of inequalities is needed to describe the stable set polytope of such graphs: constraints $F\left(Q^{\prime}\right)$ with

$$
2 x\left(Q^{\prime}\right)+x\left(N^{\prime}\left(Q^{\prime}\right)\right) \leq 2
$$

where

$$
N^{\prime}\left(Q^{\prime}\right)=\left\{v \in V(G): Q^{\prime} \subseteq N(v)\right\} .
$$

Such constraints $F\left(Q^{\prime}\right)$ are valid for all graphs $G$ with $\alpha(G) \leq 2$ and include clique constraints (if $Q^{\prime}$ is maximal and, thus, $N^{\prime}\left(Q^{\prime}\right)=\emptyset$ holds). Furthermore, Cook (see [17]) showed that $F\left(Q^{\prime}\right)$ is a facet if and only if $\bar{G}\left[N^{\prime}\left(Q^{\prime}\right)\right]$ has no bipartite component.

We call the constraints $F\left(Q^{\prime}\right)$ clique-neighborhood constraints as $N^{\prime}\left(Q^{\prime}\right)=$ $\left\{v \in V(G): Q^{\prime} \subseteq N(v)\right\}$, and conclude that the stable set polytope of a pseudothreshold graph $G=(S \cup Q, E)$ is described by nonnegativity and cliqueneighborhood constraints only.

If both parts $S$ and $U$ are non-empty, then $Q$ is a clique cutset of a pseudothreshold graph $G=(S \cup Q \cup U, E)$ due to Theorem 3, since $Q$ is a clique whose removal disconnects the graph. Hence, we can apply Chvátal's result from [7] that the facets of $\operatorname{STAB}(G)$ belong to the union of the facets of $\operatorname{STAB}(G[S \cup Q])$ and $\operatorname{STAB}(G[U \cup Q])$.

Based on these results, we can prove:

Theorem 6 The stable set polytope of pseudothreshold graphs is given by nonnegativity and clique-neighborhood constraints only.

In order to verify the $L S_{+}$-Perfect Graph Conjecture for pseudothreshold graphs, we further determine when a clique-neighborhood constraint defines a facet of the stable set polytope of a pseudothreshold graph and use a result on $L S_{+}$-perfect graphs with $\alpha(G) \leq 2$ from [2].

We next describe precisely those clique-neighborhood constraints which define facets for pseudothreshold graphs. Given a graph $G=(V, E)$, we define a block $B$ to be an inclusion-wise maximal subset of nodes such that each node in $V-B$ is adjacent to all nodes in $B$. Clearly, $G$ is the complete join of its blocks (therefore, blocks are sometimes also called anticomponents). If $G$ has stability number $\alpha(G)=2$, then a block is imperfect if and only if it contains an odd antihole by [5], see Figure 2 for illustration. A clique-neighborhood constraint $F\left(Q^{\prime}\right)$ defines a facet if and only if $\bar{G}\left[N^{\prime}\left(Q^{\prime}\right)\right]$ has no bipartite component, i.e., if each block of $G\left[N^{\prime}\left(Q^{\prime}\right)\right]$ has an odd antihole.

Based on these results, we can prove: 
Theorem 7 Let $G=(S \cup Q \cup U, E)$ be a pseudothreshold graph. A cliqueneighborhood constraint $F\left(Q^{\prime}\right)$ defines a facet of $\operatorname{STAB}(G)$ if and only if

- $F\left(Q^{\prime}\right)$ is a clique constraint $x\left(Q^{\prime}\right) \leq 1$ where $Q^{\prime}$ equals $N[v]$ for some node $v \in S$ or is a maximal clique in $G[U \cup Q]$,

- $F\left(Q^{\prime}\right)$ is a constraint $2 x\left(Q^{\prime}\right)+x\left(N^{\prime}\left(Q^{\prime}\right)\right) \leq 2$ where $N^{\prime}\left(Q^{\prime}\right)$ consists of some imperfect blocks of $G[U], Q^{\prime}$ is a maximal clique in $\left(U-N^{\prime}\left(Q^{\prime}\right)\right) \cup Q$,

- $F\left(Q^{\prime}\right)$ is the rank constraint $x(U) \leq 2$ associated with $Q^{\prime}=\emptyset$ if $Q=\emptyset$ and every block of $G[U]$ is imperfect.

In [2], it was proved that all facet-defining $L S_{+}$-perfect graphs $G$ with $\alpha(G)=2$ are odd antiholes or complete joins of one or several odd antihole(s) and a (possibly empty) clique.

Both results together imply that, in an $L S_{+}$-perfect pseudothreshold graph, a clique-neighborhood constraint $F\left(Q^{\prime}\right)$ defines a non-clique facet if and only if $N^{\prime}\left(Q^{\prime}\right)$ equals one or the complete join of some odd antiholes, and we obtain:

Corollary 8 A pseudothreshold graph is joined a-perfect if and only if every imperfect block of $G[U]$ equals an odd antihole.

To illustrate this with the help of an example, consider the pseudothreshold graph $G$ depicted in Figure 2. Within $G[U]$, there are two blocks: $B_{1}$ induces a $C_{5}$, but $B_{2}$ is different from an odd antihole, thus $G$ is not joined a-perfect. However, removing node $v_{11}$ yields a pseudothreshold graph with the property that every imperfect block of $G[U]$ equals an odd antihole and is, thus, joined a-perfect.

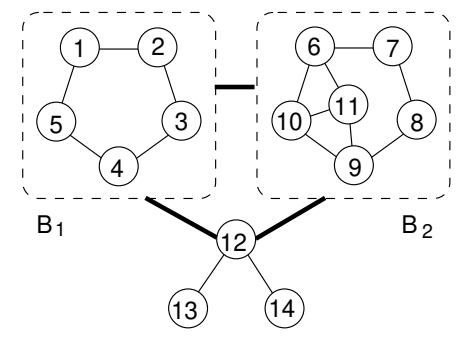

Figure 2: An example of a pseudothreshold graph: The two nodes $v_{14}$ and $v_{13}$ constitute the stable set $S$, the node $v_{12}$ the clique $Q$, and all nodes $v_{1} \ldots v_{11}$ belong to $U$. Within $G[U]$, there are two blocks: nodes $v_{1} \ldots v_{5}$ constitute one block $B_{1}$ and induce a $C_{5}$, nodes $v_{6} \ldots v_{11}$ constitute a second block $B_{2}$ and induce another imperfect graph. The presence of all possible edges between $B_{1}$ and $B_{2}$ as well as between the clique $Q$ and $B_{1}, B_{2}$ is indicated by bold lines.

Corollary 8 further implies:

Corollary 9 The $L S_{+}$-Perfect Graph Conjecture is true for pseudothreshold graphs. 
It is left to draw some conclusions for subclasses of pseudothreshold graphs. Recall that a graph $G$ is strongly pseudothreshold if both $G$ and $\bar{G}$ are pseudothreshold. Due to Theorem 3, it is easy to see that $S$ and $Q$ change their roles in $\bar{G}$ such that $\bar{G}$ is pseudothreshold if and only if also $\bar{G}[U]$ has stability number 2 . We can characterize strongly pseudothreshold graphs as follows:

Theorem 10 A graph $G=(S \cup Q \cup U, E)$ is strongly pseudothreshold if and only if $G[U]$ is empty or equals $C_{4}, \bar{C}_{4}$, or $C_{5}$.

Due to Theorem 7 and Theorem 10, in the stable set polytope of a strongly pseudothreshold graph $G=(S \cup Q \cup U, E)$, the only non-clique facet can be

$$
2 x(Q)+x(U) \leq 2
$$

if $G[U]=C_{5}$, which is a joined antiweb constraint. Hence, we conclude:

Corollary 11 Strongly pseudothreshold graphs are joined a-perfect.

Note that this result includes the subclass of $\left(C_{4}, \bar{C}_{4}\right)$-free graphs, which are obviously strongly pseudothreshold by Theorem 10 and the result of Blázsik et al. [3] showing that $\left(C_{4}, \bar{C}_{4}\right)$-free graphs are precisely the pseudothreshold graphs with $U=\emptyset$ or $U=C_{5}$.

\section{About the graphs in $\mathcal{G}_{U}$}

In order to describe the facet-defining system of inequalities of the stable set polytope for graphs $G$ in $\mathcal{G}_{U}$, we rely on the decomposition theorem by Boncompagni et al. [4] (see Theorem 5) telling that $G$ either has a clique cutset or is a light clique or a fat universal wheel.

In the case that $G$ has a clique cutset $Q$, we know from [7] that the facets of $\operatorname{STAB}(G)$ belong to the union of the facets of the stable set polytopes of the blocks of the decomposition (i.e. to $G\left[V_{i} \cup Q\right]$ for any component $V_{i}$ of $G-Q$ ).

Any graph $G$ in $\mathcal{G}_{U}$ without a clique cutset is either a light clique (and, thus, perfect), or a fat universal wheel (and, thus, the complete join of a hole $C$ of length $k \geq 5$ and a (possibly empty) clique $Q$ ).

In the latter case, $G$ is perfect (if $C$ is even), or an odd hole (if $C$ is odd and $Q$ is empty), or else (if $C$ is odd and $Q$ non-empty) defines the facet

$$
x(C)+\alpha(C) x(Q) \leq \alpha(C)=\frac{|C|-1}{2}
$$

by the behavior of the stable set polytope under taking complete joins due to Chvátal [7].

Calling such inequalities fat universal wheel constraints, we conclude: 
Theorem 12 The stable set polytope of graphs in $\mathcal{G}_{U}$ is completely described by

- nonnegativity constraints,

- clique constraints,

- odd hole constraints,

- fat universal wheel constraints.

As constraints associated with cliques, odd holes or fat universal wheels are clearly special cases of joined antiweb constraints, we conclude:

Corollary 13 All graphs in $\mathcal{G}_{U}$ are joined a-perfect.

A further consequence is concerned with universally signable graphs. Recall that a universally signable graph is either a clique, a hole, or has a clique cutset (Theorem 4).

Since every clique is in particular a light clique (where the matching is empty) and every hole is a special fat universal wheel (where the involved clique is empty), we clearly have that every universally signable graph belongs to $\mathcal{G}_{U}$. We further conclude from Theorem 12:

Corollary 14 The stable set polytope of every universally signable graph is given by nonnegativity, clique and odd hole constraints. Every universally signable graph is h-perfect.

\section{Conclusion and future research}

The context of this work was the study of $L S_{+}$-perfect graphs, i.e., graphs where a single application of the Lovász-Schrijver PSD-operator $L S_{+}$to the edge relaxation yields the stable set polytope. The $L S_{+}$-Perfect Graph Conjecture says that such graphs precisely coincide with joined a-perfect graphs.

In this work, we identified subclasses of joined a-perfect graphs: strongly pseudothreshold graphs, universally signable graphs, and $\mathcal{G}_{U}$. Whereas it follows directly from Theorem 4 and Theorem 5 that universally signable graphs form a subclass of $\mathcal{G}_{U}$, we further establish:

Theorem 15 Every strongly pseudothreshold graph belongs to $\mathcal{G}_{U}$.

Indeed, by Theorem 10, every strongly pseudothreshold graph $G=(S \cup Q \cup$ $U, E)$ has $Q$ as clique cutset and the blocks of the decomposition are light cliques (if $G[U]$ is empty or equals $C_{4}, \bar{C}_{4}$ ) or a fat universal wheel (if $G[U]$ equals $C_{5}$ ). Hence, also graphs without $C_{4}, \bar{C}_{4}$ form a subclass of $\mathcal{G}_{U}$ by [3]. Note however that the universal 5 -wheel is strongly pseudothreshold but not universally signable, whereas $C_{7}$ is universally signable but not strongly pseudothreshold.

Moreover, we note that $\mathcal{G}_{U}$ forms a new subclass of joined a-perfect graphs, since $\mathcal{G}_{U}$ is incomparable to all previously known such classes:

- $\bar{C}_{6}$ is t-perfect (and thus also h-perfect and a-perfect) as well as nearbipartite, but belongs clearly not to $\mathcal{G}_{U}$ (recall that $\bar{C}_{6}$ is a prism); 
- every strongly pseudothreshold graph $G=(S \cup Q \cup U, E)$ with $G[U]=C_{5}$ and $S \neq \emptyset$ is not near-bipartite (as the non-neighbors of any node in $S$ contain $C_{5}$ ).

Note further that $\mathcal{G}_{U}$ is a proper subclass of joined a-perfect graphs (for instance $\bar{C}_{7}$ is joined a-perfect but not in $\mathcal{G}_{U}$ by Theorem 5 by [4]).

In addition, we verified the $L S_{+}$-Perfect Graph Conjecture for pseudothreshold graphs. We shortly discuss the conjecture for a superclass $\mathcal{G}_{U T}$ of $\mathcal{G}_{U}$, defined in [4] as the class of all graphs not having thetas, pyramids, prisms and proper wheels.

By definition, we have for any graph $G$ in $\mathcal{G}_{U T}$ that $G$ either belongs to $\mathcal{G}_{U}$ or else has a twin wheel (i.e. a hole $C$ and an additional node $v$ that is adjacent to precisely 3 consecutive nodes of $C$ ). From results in [15], it follows that a twin wheel is $L S_{+}$-imperfect whenever $C$ is odd: the twin wheel with $C=C_{5}$ was identified as $L S_{+}$-imperfect graph in [15]. In addition, there it was proven that further $L S_{+}$-imperfect graphs can be obtained by applying certain operations preserving $L S_{+}$-imperfection, including the even subdivision of edges. Clearly, any twin wheel with $C=C_{2 k+1}$ is an even subdivision of the twin wheel with $C=C_{5}$ so that all twin wheels with odd $C$ are $L S_{+}$-imperfect.

This shows that a graph in $\mathcal{G}_{U T}$ can be $L S_{+}$-perfect only if it has no odd twin wheel. Though every even twin wheel is perfect, this does not yet verify the $L S_{+}$-Perfect Graph Conjecture for $\mathcal{G}_{U T}$ : for instance the graph obtained from $\bar{C}_{7}$ by replicating one node is a graph in $\mathcal{G}_{U T}$ without an odd twin wheel (but containing a twin 4 -wheel), but is $L S_{+}$-imperfect by [1].

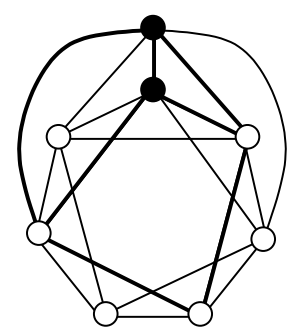

Figure 3: The graph obtained from $\bar{C}_{7}$ by replicating one node, the contained twin 4-wheel is highlighted by bold edges.

Hence, it remains open to verify the $L S_{+}$-Perfect Graph Conjecture for $\mathcal{G}_{U T}$. This could be done with the help of a decomposition theorem for $\mathcal{G}_{U T}$ presented in [4], provided that the facet-defining system of the stable set polytope for all basic classes of $\mathcal{G}_{U T}$ can be found.

Finally, we note that the approach presented here to verify the $L S_{+}$-Perfect Graph Conjecture can be applied to all graph classes whose members can be decomposed along clique cutsets into basic classes for which the stable set polytope can be completely described. 


\section{References}

[1] S. Bianchi, M. Escalante, G. Nasini, and L. Tunçel. Lovász-Schrijver PSDoperator and a superclass of near-perfect graphs. Electronic Notes in Discrete Math., 44:339-344, 2013.

[2] S. Bianchi, M. Escalante, G. Nasini, and A. Wagler. Lovász-Schrijver PSDoperator on claw-free graphs. In R. Cerulli, S. Fujishige, and R. Mahjoub, editors, Combinatorial Optimization (ISCO 2016), pages 59-70, Cham, 2016, Springer.

[3] Z. Blázsik, M. Hujter, A. Pluhár, and Z. Tuza. Graphs with no induced $C_{4}$ and $2 K_{2}$. Discrete Math., 115:51-55, 1993.

[4] V. Boncompagni, I. Penev, and K. Vuşković. Clique-cutsets beyond chordal graphs, arXiv:1707.03252 [math.CO].

[5] M. Chudnovsky, N. Robertson, P. Seymour, and R. Thomas. The strong perfect graph theorem. Annals of Mathematics, 164:51-229, 2006.

[6] V. Chvátal. Edmonds polytopes and a hierarchy of combinatorial problems. Discrete Math., 4:305-337, 1973.

[7] V. Chvátal. On certain polytopes associated with graphs. J. Combin. Theory (B), 18:138-154, 1975.

[8] V. Chvátal and P.L. Hammer. Aggregation of inequalities in integer programming. Annals of Discrete Math., 1:145-162, 1977.

[9] M. Conforti, G. Cornuéjols, A. Kapoor, and K. Vuşković. Universally signable graphs. Combinatorica, 17:67-77, 1997.

[10] S. Coulonges, A. Pêcher, and A. Wagler. Characterizing and bounding the imperfection ratio for some classes of graphs. Math. Progr. A, 118:37-46, 2009.

[11] G.A. Dirac. On rigid circuit graphs. Abhandlungen aus dem Mathematischen Seminar der Universität Hamburg, 25:71-76, 1961.

[12] M. Escalante and G. Nasini. Lovász and Schrijver $N_{+}$-relaxation on web graphs. Lecture Notes in Computer Sciences, 8596:221-229, 2014.

[13] M. Escalante, G. Nasini, and A. Wagler. Characterizing $N_{+}$-perfect line graphs. International Transactions in Operational Research, 24:325-337, 2017.

[14] S. Földes and P. Hammer. Split graphs having Dilworth number two. Canadian Journal of Mathematics, 29:666-672, 1977.

[15] L. Lipták and L. Tunçel. Stable set problem and the lift-and-project ranks of graphs. Math. Programming A, 98:319-353, 2003.

[16] L. Lovász and A. Schrijver. Cones of matrices and set-functions and 0-1 optimization. SIAM J. on Optimization, 1:166-190, 1991.

[17] F.B. Shepherd. Applying Lehman's theorem to packing problems. Mathematical Programming, 71:353-367, 1995.

[18] K. Truemper. Alpha-balanced graphs and matrices and GF(3)-representability of matroids. Journal of Combin. Theory B, 32:112-139, 1982.

[19] A. Wagler. Antiwebs are rank-perfect. 4OR, 2:149-152, 2004. 